\title{
Skeletons of stable maps II: superabundant geometries
}

Dhruv Ranganathan*

*Correspondence:

dhruvr@mit.edu

Department of Mathematics,

Massachusetts Institute of

Technology, 77 Massachusetts

Avenue, Cambridge, MA 02138 USA

\begin{abstract}
We implement new techniques involving Artin fans to study the realizability of tropical stable maps in superabundant combinatorial types. Our approach is to understand the skeleton of a fundamental object in logarithmic Gromov-Witten theory - the stack of prestable maps to the Artin fan. This is used to examine the structure of the locus of realizable tropical curves and derive three principal consequences. First, we prove a realizability theorem for limits of families of tropical stable maps. Second, we extend the sufficiency of Speyer's well-spacedness condition to the case of curves with good reduction. Finally, we demonstrate the existence of liftable genus 1 superabundant tropical curves that violate the well-spacedness condition.
\end{abstract}

\section{Background}

Central to the application of tropical techniques to questions in algebraic geometry are so-called lifting theorems. Given a "synthetic" tropical object, such as a weighted balanced polyhedral complex, one must understand whether this object is the tropicalization of an algebraic variety. We deal in this paper with the case of curves. The tropical lifting question in this setting asks, when does an embedded tropical curve in $\mathbb{R}^{n}$ arise as the tropicalization of an algebraic curve in a torus over a nonarchimedean field? This question becomes highly nontrivial in the so-called superabundant case and has been the primary obstacle to the application of tropical curve counting techniques in high genus settings. A tropical curve in $\mathbb{R}^{n}$ encodes the combinatorial data in a degenerate logarithmic stable map to a toric variety. If the tropical curve is superabundant, i.e., if the tropical deformation space is larger than expected, the obstruction group of this degenerate logarithmic map is nonzero. As a result, such a map may not deform and the tropical curve may fail to be realizable. See Sect. 2.2 for a precise definition of superabundance. The earliest realization theorems for superabundant curves are due to Speyer, who observed a subtle combinatorial condition guaranteeing the realizability of superabundant genus 1 tropical curves [31]. While there has been substantial additional work in the intervening years, the general question remains mysterious $[7,15,19,23-25,29,34]$.

In this note, we use recent technical breakthroughs in nonarchimedean geometry and the theory of logarithmic maps to provide a conceptual framework in which the realizability question may be approached. To demonstrate the efficacy of this framework, we use it to give simple proofs of three new results for lifting tropical curves. The same frame-

(c) The Author(s) 2017. This article is distributed under the terms of the Creative Commons Attribution 4.0 International License (http://creativecommons.org/licenses/by/4.0/), which permits unrestricted use, distribution, and reproduction in any medium, provided you give appropriate credit to the original author(s) and the source, provide a link to the Creative Commons license, and indicate if changes were made. 
work provides new insight into the structure of realizability conditions more globally-the locus of realizable tropical curves is given by a union of bend loci of a collection of tropical polynomials in the edge lengths of the tropical curve.

\subsection{Statement of results}

All valued fields appearing in this paper will have equicharacteristic zero. Throughout, the symbol $\square$ will be used to denote an abstract tropical curve. ${ }^{1}$ We say that a parameterized tropical curve $\left[\left[\rightarrow \mathbb{R}^{n}\right]\right.$ is realizable if there exists a smooth curve $C$ over a nonarchimedean field and a map $\left[C \rightarrow \mathbb{G}_{m}^{n}\right]$ whose tropicalization is $\left[\left[\rightarrow \mathbb{R}^{n}\right]\right.$.

Theorem A Let $\left[\complement_{t} \rightarrow \mathbb{R}^{n}\right]$ for $t \in[0,1)$ be a continuously varying family of parameterized tropical curves. Let $\left[\square_{1} \rightarrow \mathbb{R}^{n}\right]$ denote the limit of this family in the moduli space of parameterized tropical curves. If $\left[\square_{t} \rightarrow \mathbb{R}^{n}\right]$ is realizable for all $t \in[0,1)$, then the limiting map $\left[\complement_{1} \rightarrow \mathbb{R}^{n}\right]$ is realizable.

Our next result extends the reach of Speyer's well-spacedness condition to the case of elliptic curves with good stable reduction, see Definition 3.2. Let $\square$ be a tropical curve of genus 1 with a unique genus 1 vertex, so in particular the underlying graph of $\square$ is a tree. Denote by $\hat{\complement}$ the tropical curve obtained by first replacing the genus 1 vertex with a genus 0 vertex and then adding a self-loop of length 1 at $v$.

Theorem B Let $\left[\square \rightarrow \mathbb{R}^{n}\right]$ be a parameterized tropical curve of genus 1 with a unique genus 1 vertex $v$. Assume that the star of $v$ in $\left[\hat{[} \rightarrow \mathbb{R}^{r}\right]$ is realizable. If $\left[\square \rightarrow \mathbb{R}^{n}\right]$ is well-spaced, then $\left[\left[\rightarrow \mathbb{R}^{n}\right]\right.$ is realizable.

The work of Speyer shows that the well-spacedness condition is sufficient for realizability of tropical genus 1 curves. He also proves that this condition is also necessary, with the restriction that the curve is trivalent. The following result shows that outside the trivalent case, well-spacedness can be violated. This complements Baker, Payne, and Rabinoff's generalization of Speyer's condition [7, Theorem 6.9].

Theorem C Let $n \geq 3$. There exist superabundant parameterized genus 1 tropical curves $\left[\left[\rightarrow \mathbb{R}^{n}\right]\right.$ that lift to algebraic curves but violate the well-spacedness condition.

The new point of view taken in this paper is to attempt to understand the realizability locus inside the moduli space of all parameterized tropical curves as a global tropical geometric object. We do so by studying a fundamental object in logarithmic GromovWitten theory-the space of logarithmic prestable maps to the Artin fan. This is inspired by the insights of Abramovich-Wise, Gross-Siebert, and Ulirsch. By synthesizing these ideas, we are led to the following result.

Let $X$ be a toric variety with fan $\Delta$, and let $\mathscr{L}_{\Gamma}^{\circ}(X)$ denote the moduli space of maps from smooth pointed genus $g$ curves into $X$ with fixed contact orders with the toric boundary along smooth marked points. In Sect. 2.2 a generalized extended cone complex $T_{\Gamma}(\Delta)$ is constructed which parameterizes tropical stable maps with the analogous discrete

\footnotetext{
${ }^{1}$ We use $\mathscr{C}, C$, and $[$ to denote families of curves, single curves and tropical curves, respectively, choosing notation that best approximates the shape of these objects as found in the wild. We thank Dan Abramovich for this most creative of suggestions.
} 
data. The generalized cone complex $T_{\Gamma}^{\circ}(\Delta)$ is the complement of the extended faces and parameterizes maps from tropical curves where all edge lengths are finite.

\section{Theorem D There is a continuous tropicalization map}

$$
\text { trop : } \mathscr{L}_{\Gamma}^{\circ}(X)^{\text {an }} \rightarrow T_{\Gamma}^{\circ}(\Delta),
$$

compatible with evaluation maps and forgetful maps to the moduli space of curves. The locus in $T_{\Gamma}^{\circ}(X)$ parameterizing the set of realizable tropical curves is a closed polyhedral set. If $\sigma^{\circ} \in T_{\Gamma}^{\circ}(X)$ is the relative interior of a cell, after passing to a finite cover of $\sigma^{\circ}$ by the interior of a cone $\tilde{\sigma}^{\circ}$, the locus of realizable curves in $\tilde{\sigma}^{\circ}$ is the union of bend loci of collections of tropical polynomials.

\subsection{Further discussion}

A number of experts have made the informal observation that the condition appearing in Speyer's realizability theorem-that the minimum of a collection of numbers occurs at least twice-resembles the tropical variety of a tropical ideal. We view Theorem D as giving a simple and rigorous explanation for this phenomenon.

While the proof of our lifting theorems only relies on compactness of the realizable locus, the tropical structure is useful for applications to enumerative geometry. This is illustrated, for instance, by the results of Len and the author [20], in which the polyhedral structure of the realizability locus is used to derive multiplicities for tropical curve counting.

The above Theorem D also contributes to the study of tropical moduli spaces, which have received considerable interest in recent years. These results have aimed at an improved conceptual understanding of information contained in tropical moduli spaces, including applications to enumerative geometry $[10,11,28]$ and to the geometry and topology of moduli spaces $[9,12,13]$. The novelty of the present paper is that the tropicalization of $\mathscr{L}_{\Gamma}^{\circ}(X)$ is studied as the tropicalization of a map to a certain toroidal stack - the stack of prestable maps to the Artin fan. This is in sharp contrast to recent results on tropicalizations for moduli spaces, which have used toroidal structures on the spaces themselves.

The well-spacedness condition has inspired a great deal of research. However, to our knowledge, the results of Baker-Payne-Rabinoff [7, Theorem 6.9] are the only known nontrivial necessary conditions for realizability for nonmaximally degenerate tropical curves, although it may be possible to extract such conditions using the methods of [19]. By studying the limits of nonsuperabundant families of curves and applying Theorem A, one can obtain sufficient conditions for lifting tropical curves in nonmaximally degenerate situations, i.e., when vertices carry nonzero genus. Furthermore, Theorem B exhibits the first instance of a sufficient condition for the realizability for nonmaximally degenerate superabundant curves.

In addition to the work of Baker-Payne-Rabinoff mentioned above, Katz extracts a number of necessary conditions for realizability. These stem from interpreting the logarithmic tangent-obstruction complex for maps to toric varieties combinatorially for degenerate maps [19]. A similar approach is used by Cheung, Fantini, Park, and Ulirsch to prove that in a large range of cases, nonsuperabundance is a sufficient condition for realizability [15]. Note, however, that limits of nonsuperabundant tropical curves can often become superabundant. As a result, Theorem A extends the reach of these theorems as well. 
An implicit goal of this paper is to demonstrate the usefulness of the perspective on tropicalization arising from logarithmic prestable maps to Artin fans arising from the work of Abramovich, Ulirsch, and Wise [5,37,38] and the insights of Gross and Siebert [17]. Indeed, accepting this technical input, the reader will note that the proofs of our realizability theorems follow from reductions to existing theorems in tropical geometry.

The first part of this project [28], which was a chapter in author's doctoral dissertation, studies the tropicalization of the moduli space of logarithmic maps to toric varieties in genus 0 , and it is in this sense that the present paper is a sequel. Superabundance never appears in the genus 0 setting, and the analogue of Theorem $\mathrm{D}$ can be used to derive a number of consequences concerning the geometry of the space of maps. We refer to loc. cit. for details. We also note that a similar polyhedrality result as above has been proved by Tony $\mathrm{Yu}$ in the context of nonarchimedean analytic Gromov-Witten theory using methods that are quite different from ours $[40,41]$.

Remark 1.1 In the months between when the first version of this paper appeared on ar $\chi$ iv and the final publication, there has been additional progress on tropical realizability. The framework established here has been used by Jensen and the author to prove realization theorems for superabundant tropical curves in the "chain of cycles" combinatorial type, in arbitrary genus. This has in turn found applications in Brill-Noether theory, see [18, Theorems A \& B].

\section{Prerequisites}

We assume familiarity with the fundamental concepts of Berkovich geometry and logarithmic structures. A rapid overview of the relevant concepts may be found in the preceding article [28, Section 2]. We refer the reader to two excellent recent surveys in this area by Abramovich, Chen, and their collaborators $[3,4]$.

\section{Tropicalization for maps and their moduli}

\subsection{Logarithmic stable maps}

Our central object of study is the moduli space of genus $g$ curves in a projective toric variety $X$ in a fixed curve class, meeting each torus invariant divisor $D_{\rho}$ at marked points with prescribed contact orders. We work with the compactification of this space of ramified curves, provided by the Abramovich-Chen-Gross-Siebert theory of logarithmic stable maps $[2,14,17]$.

Let $X$ be a projective toric variety with dense torus $T$, corresponding to a fan $\Delta$ in the vector space $N_{\mathbb{R}}$ spanned by the cocharacters of $T$. Fix integers $g$ and $n$. We study the moduli space $\mathscr{L}(X)$ of families of minimal stable logarithmic morphisms

$$
f:\left(C, p_{1}, \ldots, p_{n}\right) \rightarrow X,
$$

from nodal pointed genus $g$ curves $\left(C, p_{1}, \ldots, p_{n}\right)$.

Remark 2.1 Minimality is a condition on the characteristic monoids of the logarithmic structure on the base of the family of maps. The reader may think about this concept as follows. A logarithmic stable map $[f: C \rightarrow X]$ is a stable map in the category of logarithmic schemes. Consequently, given a logarithmic scheme $S$, the moduli problem returns the collection of isomorphism classes of logarithmic stable maps over the base logarithmic scheme $S$. However, algebraic stacks are defined over the category of schemes rather than 
logarithmic schemes. A crucial and beautiful technical achievement of Abramovich, Chen, Gross, and Siebert was to identify an algebraic stack $\mathscr{L}(X)$ that represented a logarithmic moduli problem - the moduli problem for minimal maps. Given a scheme $\underline{S}$ without a chosen logarithmic structure and a map $\underline{S} \rightarrow \mathscr{L}(X)$, there is a minimal logarithmic structure $\mathscr{M}_{S}$ that can be placed on $\underline{S}$, returning a diagram in the logarithmic category

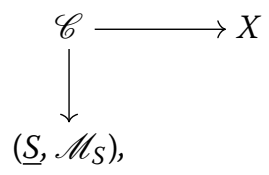

which is a family of logarithmic stable maps. Families obtained by such logarithmic structures are referred to as minimal logarithmic stable maps. The algebraic stack $\mathscr{L}(X)$ carries a universal logarithmic structure, and it is by pulling back this structure that we obtain the minimal logarithmic structure $\mathscr{M}_{S}$ as above. One can thus interpret this moduli space $\mathscr{L}(X)$ as a parameter space for minimal logarithmic stable maps and thus as a stack over the category of schemes. Given a different logarithmic structure $\mathscr{M}_{S}^{\prime}$ on $\underline{S}$, the universal property of minimality ensures that any map $\left(\underline{S}, \mathscr{M}_{S}^{\prime}\right) \rightarrow \mathscr{L}(X)$ factors uniquely through a minimal family. We refer the reader to [17, Section 1] for an explicit description of these monoids and to [2, Section 2] for a conceptual discussion.

Stability for logarithmic maps amounts to stability of the underlying map-all contracted rational components must have at least 3 special points, and all contracted elliptic components must have at least 1 special point. We will also fix the contact orders on $C$. The contact order will be recorded by a function

$$
c:\left\{p_{1}, \ldots, p_{n}\right\} \rightarrow \Delta(\mathbb{N}),
$$

where $\Delta(\mathbb{N})$ denotes the integral points of the fan $\Delta$. If $c\left(p_{i}\right)=e \cdot v_{\rho}$ where $v_{\rho}$ is a primitive generator of the ray corresponding to $D_{\rho}$, then the curve has contact order $e$ along $D_{\rho}$ at $p_{i}$. Note that by Fulton-Sturmfels' description of the Chow cohomology of a complete toric variety as Minkowski weights [16], the data of $\boldsymbol{c}$ determine an operational curve class $f_{\star}[C] \in A_{1}(X ; \mathbb{Z})$.

We package the discrete data by the symbol $\Gamma=(g, n, c)$. Let $\mathscr{L}_{\Gamma}(X)$ denote the moduli space of minimal logarithmic maps carrying discrete data $\Gamma$, and let $\mathscr{L}_{\Gamma}^{\circ}(X)$ be the locus on which the logarithmic structure is trivial. The following result is established by Abramovich-Chen [2, Section 5] and Gross-Siebert [17, Corollary 4.2].

Theorem 2.2 The moduli space $\mathscr{L}_{\Gamma}(X)$ of minimal logarithmic stable maps with discrete data $\Gamma$ is a proper logarithmic algebraic stack with projective coarse moduli space.

Note that in this paper, we will often work with logarithmic maps over valuation rings $\operatorname{Spec}(R)$ that are not discretely valued. The natural logarithmic structure in this case is coherent, but not fine. In this case, one may approximate $R$ by sub-DVR's and pass to a limit, see [5, Appendix A.1]. Alternatively, in Theorem A, one can pass to a subsequence of $[0,1)$ converging to 1 , parameterizing tropical maps with rational edge lengths. This will eliminate the need to work with maps over nondiscrete valuation rings in the sequel. 


\subsection{Tropical stable maps}

The purpose of this section is to construct a parameter space for tropical stable maps, which will serve as the target of our tropicalization map.

Definition 2.3 A prestable $n$-pointed tropical curve of genus $g$, denoted $\sqsubset$, is a finite graph $G(\sqsubset)$ and three additional data:

(1) To each edge of $e \in G(\sqsubset)$, a length $\ell(e) \in \mathbb{R}_{\geq 0} \sqcup\{\infty\}$ such that if $e$ is a marked leaf edge, $\ell(e)=\infty$.

(2) To every vertex $v \in G(\sqsubset)$ a genus $g(v) \in \mathbb{Z}_{\geq 0}$.

(3) A labeling of a subset of the 1 -valent genus 0 vertices of $\sqsubset$ by the set $\left\{p_{1}, \ldots, p_{n}\right\}$.

The genus of $\square$ is defined to be

$$
g(\sqsubset)=h^{1}(\sqsubset)+\sum_{v \in \sqsubset} g(v) .
$$

The tropical curve $\square$ is made into a topological space as follows. Each edge $e$ of finite length $\ell(e)$ is identified with $[0, \ell(e)]$. Marked leaf edges are metrized as $[0, \infty]$. Unmarked edges $e$ with $\ell(e)=\infty$ are topologized as two glued intervals $[0, \infty] \sqcup_{\infty}[0, \infty]$. We will refer to a curve $[$ whose lengths are finite away from the marked leaves as smooth.

Informally, $\square$ is often thought of as being a metric space away from the infinite points and as a space with a "singular" metric when including the infinite points.

Remark 2.4 The terminology of "smooth" here is motivated by the following fact. Given a tropical curve $[$, one can consider any family $\mathscr{C}$ of marked prestable curves over a valuation ring $\operatorname{Spec}(R)$, such that the skeleton of $\mathscr{C}$ is $\sqsubset$. The general fiber $\mathscr{C}_{\eta}$ of this family is smooth if and only if the nonleaf edge lengths of $\square$ are all finite, i.e., if $\square$ is smooth in the above sense. To see this, consider an edge $e$ of $\sqsubset$ corresponding to a node $q$ of $\mathscr{C}_{0}$. Formally locally near $q$, the total family may be described as $x y=f$, where $f \in R$, and the valuation of $f$ is identified with the length of $e$. The valuation of $f$ is infinity if and only if $f$ is zero. In turn $f$ is zero if and only if the node $q$ persists in the generic fiber of $\mathscr{C}$, see $[1,7]$.

Recall that a morphism $\phi: \Sigma_{1} \rightarrow \Sigma_{2}$ between polyhedral complexes is a map on the underlying point sets such that each polyhedron in $\Sigma_{1}$ is mapped to a polyhedron in $\Sigma_{2}$.

Definition 2.5 A tropical stable map from a smooth tropical curve is a continuous and proper morphism of polyhedral complexes

$$
f:\left(\sqsubset,\left\{p_{1}, \ldots, p_{n}\right\}\right) \rightarrow(\bar{\Delta}, \partial \Delta)
$$

where $\left(\sqsubset,\left\{p_{1}, \ldots, p_{n}\right\}\right)$ is a smooth $n$-marked abstract tropical curve, such that the following conditions are satisfied.

(TSM1) For each edge $e \in \llbracket$, the direction of $f(e)$ is an integral vector. Moreover, upon restriction to $e, f$ has integral slope $w_{e}$, taken with respect to this integral direction. This integral slope is referred to as the expansion factor of $f$ along $e$.

(TSM2) The map $f$ is balanced in the usual sense, i.e., at all points of $\square$ the sum of the derivatives of $f$ in each tangent direction is zero.

(TSM3) The map $f$ is stable. That is, if $p \in[$ has valence 2, then the image of $\operatorname{Star}(v)$ is not contained in the relative interior of a single cone of $\Delta$. 
We will usually suppress the markings from the notation and simply write the map as $[\sqsubset \rightarrow \bar{\Delta}]$.

The following definition indexes the "deformation class" of a tropical stable map and is obtained by dropping the data of the lengths of the edges of $\sqsubset$.

Definition 2.6 The combinatorial type of a tropical stable map $[[\rightarrow \bar{\Delta}]$ is the data

(CT1) The finite graph model $G(\sqsubset)$ underlying $\sqsubset$.

(CT2) For each vertex $v \in G(\sqsubset)$, a cone $\sigma_{v} \in \Delta$ containing the image of $v$.

(CT3) For each edge $e$, the slope $w_{e}$ of $f$ restricted to $e$ and the primitive vector $u_{e}$ in the direction of $f(e)$.

Definition 2.7 The recession type of a combinatorial type $\Theta$ is obtained from $[\square \rightarrow \bar{\Delta}]$ by collapsing all bounded edges of $\square$ to a single vertex. That is, $\square$ is a single vertex with genus $g$ and marked edges, and the marked edges are each decorated by a contact order.

The following proposition seems to be well-known to experts, and a formal proof in the $g=0$ case may be found in [25, Proposition 2.1] or [28, Proposition 3.2.1]. We give an outline of the argument in general.

Proposition 2.8 Let $\Theta$ be the combinatorial type of a tropical stable map. The set of all tropical curves $[[\rightarrow \bar{\Delta}]$ together with an identification with the type $\Theta$ is parameterized by a cone $\sigma_{\Theta}$. Further, there are finitely many combinatorial types with fixed recession type.

Proof Fix a combinatorial type $\Theta=[[\rightarrow \Delta]$ and let $V$ and $E$ be the vertex and bounded edge sets of $\sqsubset$, respectively. To describe a particular tropical map $[f]$ in this combinatorial type is to assign to each $v_{i}$, a point $f\left(v_{i}\right)$ in the associated cone $\sigma_{v_{i}}$ and to each edge $e_{j}$, a real edge length $\ell_{e}$. In order that these assignments define a continuous and balanced piecewise-linear map to $\Delta$, we simply need to force that for every edge $e$ connecting to $v_{e 1}$ and $v_{e 2}$,

$$
f\left(v_{e 1}\right)-f\left(v_{e 2}\right)=\ell_{e} w_{e} .
$$

Here, in keeping with previous notation, $w_{e}$ is the vector slope prescribed by the combinatorial type. In other words, the set of tropical curves is the subcone of $\prod_{v \in V} \sigma_{v} \times \prod_{e \in E} \mathbb{R}_{\geq 0}$ given by

$$
\sigma_{\Theta}=\left\{\left(\left(f(v)_{v},\left(\ell_{e}\right)_{e}\right) \in \prod_{v \in V} \sigma_{v} \times \prod_{e \in E} \mathbb{R}_{\geq 0}: \text { For all } e=v_{e 1} v_{e 2} \in E, v_{e 1}-v_{e 2}=\ell_{e} w_{e}\right\} .\right.
$$

These relations cut out a subcone, and the result follows. The claimed finiteness follows from the boundedness of combinatorial types for logarithmic maps, as proved in [17, Section 3.1].

Following [22, Proposition 2.14], the overvalence of a type $\Theta$ is defined as

$$
\operatorname{ov}(\Theta)=\sum_{p: \operatorname{val}(p) \geq 4} \operatorname{val}(p)-3 .
$$

The expected dimension of this cone of tropical maps is

$$
\operatorname{dim} \sigma_{\Theta}=(\operatorname{dim}(\Delta)-3)\left(1-b_{1}(\sqsubset)\right)+n-\operatorname{ov}(\Theta),
$$


where $b_{1}(\sqsubset)$ is the first Betti number of the underlying graph of $\sqsubset$. This dimension calculation explained, for instance, in [25, Section 1]. Just as in the algebraic case, it can be deduced from an analysis of the combinatorial tangent-obstruction complex, i.e., the "abundancy map" in [15, Definition 4.1]. See also [22, Sections 2.4-2.6]. The expected dimension above is a lower bound for the dimension of $\sigma_{\Theta}$, but the actual dimension is often be larger. For instance, if cycles of $\square$ are mapped into proper affine subspaces of $|\Delta|$ of high codimension, the dimension of the actual deformation space will be larger than the above-expected dimension.

Definition 2.9 A combinatorial type $\Theta$ is said to be superabundant if the dimension of $\sigma_{\Theta}$ is strictly larger than the expected dimension.

Following [1, Sections 2.3-2.4] there is a natural compactification of any cone $\sigma$ with integral structure obtained as follows. Let $S_{\sigma}$ be the monoid of integral linear functions on $\sigma$ that are nonnegative, i.e., the dual monoid of $\sigma$. The cone $\sigma$ can be recovered as the space of monoid homomorphisms $\operatorname{Hom}\left(S_{\sigma}, \mathbb{R}_{\geq 0}\right)$. The canonical compactification of $\sigma$ is defined as

$$
\bar{\sigma}:=\operatorname{Hom}\left(S_{\sigma}, \mathbb{R}_{\geq 0} \sqcup\{\infty\}\right) .
$$

Fixing a combinatorial type $\Theta$, the faces of the extended cone $\bar{\sigma}_{\Theta}$ parameterize tropical stable maps where $\sqsubset$ is possibly singular and the vertices of $\square$ map to the extended faces of $\bar{\Delta}$. We will have no need to work with such maps directly, so we leave the precise formulation to an interested reader.

Definition 2.10 An isomorphism between two tropical stable maps $\left[\left[_{1} \rightarrow \Delta\right]\right.$ and $\left[\left[_{2} \rightarrow \Delta\right]\right.$ is an isometry of graphs, commuting with the vertex weights and with the map:

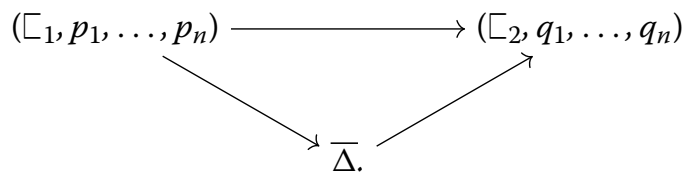

An isomorphism of a stable map with itself is said to be an automorphism. Similarly, an automorphism of the combinatorial type $\Theta$ is an automorphism of the underlying finite graph $G(\sqsubset)$ preserving the edge directions, their expansion factors, vertex weights, and the cones associated with each vertex.

The moduli cones for fixed combinatorial types described above form the local models for the space of tropical stable maps. The globalization is achieved by what are now standard techniques, introduced by Abramovich, Caporaso, and Payne [1, Section 4] for the moduli spaces of curves. In the maps setting, this can be found in [28, Section 3] in the genus 0 case. The changes in higher genus are not substantive. Given any combinatorial type $\Theta$, the faces of $\sigma_{\Theta}$ also parameterize maps from tropical curves. A moduli cone $\sigma_{\Theta^{\prime}}$ is a face of $\sigma_{\Theta}$ if and only if

(1) The source type $G\left(\complement^{\prime}\right)$ and $\Theta^{\prime}$ is obtained from the source graph $G(\sqsubset)$ of $\Theta$ by a (possibly trivial) sequence of edge contractions $\alpha: G \rightarrow G^{\prime}$.

(2) Given any vertex $v^{\prime} \in G\left(\check{\complement}^{\prime}\right)$ and a vertex $v$ such that $\alpha(v)=v^{\prime}$, then the cone $\sigma_{v^{\prime}}$ is a face of $\sigma_{v}$. 
The moduli space of tropical stable maps is defined to be

$$
T_{\Gamma}(\Delta):=\underset{\Theta: \text { stable }}{\lim }\left(\bar{\sigma}_{\Theta}, j_{\Theta}\right),
$$

where the objects of the colimit are all combinatorial types of a give recession type. The maps $j_{\Theta}$ range over all identifications of faces, as detailed above, and over automorphisms of combinatorial types. We will refer to the image of an extended cone $\bar{\sigma}_{\Theta}$ in the colimit above as a cell of the generalized cone complex.

The topological space constructed above has the structure of a generalized extended cone complex in the sense of [1, Section 2]. By forming the union of the images of the ordinary (i.e., noncompact) cones $\sigma_{\Theta}$ in $T_{\Gamma}(\Delta)$, we obtain the moduli space $T_{\Gamma}^{\circ}(\Delta)$, parameterizing those maps with smooth source graph.

\subsection{Prestable tropical maps}

For our later study, it will be convenient to relax the stability condition above. A prestable tropical map to $\Delta$ is a map [ $[\rightarrow \Delta]$ as in Definition 2.5 , but possibly violating the stability condition (TSM3). In other words, $[[\rightarrow \Delta]$ becomes a stable map after dropping finitely many 2 -valent vertices from $\sqsubset$. There are infinitely many such destabilizations of any combinatorial type. However, there are no substantive changes to the construction above-prestable tropical curves of a fixed combinatorial type are still parameterized by a cone, though of arbitrarily high dimension. Gluing these cones together exactly as above, we obtain a generalized extended cone complex

$$
T_{\Gamma}^{\text {pre }}(\Delta):=\underset{\Theta: \text { prestable }}{\lim }\left(\bar{\sigma}_{\Theta}, j_{\Theta}\right) .
$$

In analogy with toroidal embeddings that are locally of finite type, one might regard this cone complex as being locally of finite type.

The crucial technical connection between the logarithmic maps theory and tropical geometry comes from an elegant observation of Gross and Siebert which we now recall. Let $[C \rightarrow X]$ be a logarithmic stable map over a logarithmic point $\operatorname{Spec}(P \rightarrow \mathbb{C})$. As explained at the beginning of this section, a map is said to be minimal if the logarithmic structure given by $P$ on $\operatorname{Spec}(\mathbb{C})$ coincides with the logarithmic structure obtained by pulling back the structure on the moduli space $\mathscr{L}_{\Gamma}(X)$ via the underlying map

$$
\operatorname{Spec}(\mathbb{C}) \rightarrow \mathscr{L}_{\Gamma}(X)
$$

The logarithmic stable map [C $\rightarrow X]$ has a well-defined combinatorial type. The source graph $\Gamma$ is taken to be the dual graph of $C$, the vertices map to the cone $\sigma$ dual to the stratum containing the generic point of the corresponding component, and the expansion factors along edges are uniquely determined by the contact order, see [17, Section 1.4]. If $\Theta$ is the combinatorial type of $[C \rightarrow X]$, let $T_{\Theta}$ denote the corresponding moduli cone of tropical curves of this combinatorial type and let $T_{\Theta}(\mathbb{N})$ be the integral points of this cone.

Theorem 2.11 (Gross and Siebert [17, Section 1.5]) Let $[f: C \rightarrow X]$ be a logarithmic (pre)-stable map over $\operatorname{Spec}(P \rightarrow \mathbb{C})$ with combinatorial type $\Theta$. The map $[f]$ is minimal if and only if the dual monoid $\operatorname{Hom}(P, \mathbb{N})$ is isomorphic to the monoid $T_{\Theta}(\mathbb{N})$.

This result follows from the fact that minimality of the map $[f]$ can be characterized by placing constraints on the characteristic monoids of the base, in this case $\operatorname{Spec}(P \rightarrow \mathbb{C})$. As 
explained in [17, Section 1.6 \& Remark 1.21], the map forces certain "minimal constraints" that every such base monoid has to satisfy. This implies that there is a natural injective map $\operatorname{Hom}(P, \mathbb{N}) \rightarrow T_{\Theta}(\mathbb{N})$. The universal property of minimality [17, Proposition 1.24] forces that, if $P$ is minimal, the map is also surjective and thus an isomorphism.

The same result holds when $X$ replaced with its Artin fan $\mathscr{A}_{X}=[X / T]$.

\subsection{Pointwise tropicalization for logarithmic stable maps}

Our next goal is to construct a map

$$
\text { trop : } \mathscr{L}_{\Gamma}^{\circ}(X)^{\text {an }} \rightarrow T_{\Gamma}^{\circ}(\Delta)
$$

from the analytification of the space of maps from smooth curves with prescribed contact orders to the space of tropical maps.

Any point $x \in \mathscr{L}_{\Gamma}^{\circ}(X)^{\text {an }}$ may be represented by a map

$$
\operatorname{Spec}(K) \rightarrow \mathscr{L}_{\Gamma}^{\circ}(X),
$$

where $K$ is a valued field extension of $\mathbb{C}$. After a ramified base change, the existence of the compactification $\mathscr{L}_{\Gamma}(X)$ of $\mathscr{L}_{\Gamma}^{\circ}(X)$ guarantees an extension to

$$
\operatorname{Spec}(R) \rightarrow \mathscr{L}_{\Gamma}(X)
$$

where $R$ is the valuation ring of $K$. By pulling back the universal curve and universal map we obtain a diagram

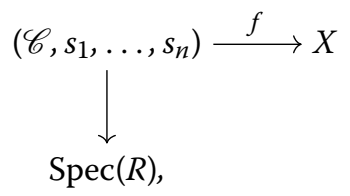

where the $s_{i}$ are horizontal sections determining marked points on the geometric fibers. By [6, Section 1.4], this choice of model determines a retraction of the analytic generic fiber onto a tropical curve

$$
\mathscr{C}_{\eta}^{\mathrm{an}} \rightarrow \sqsubset,
$$

with a canonical continuous section

$$
\sqsubset \rightarrow \mathscr{C}_{\eta}^{\text {an }} .
$$

Similarly, there is a deformation retraction

$$
X^{\text {an }} \rightarrow \bar{\Delta},
$$

of the analytic toric variety onto its skeleton by $[26,33]$. Composing the section map with the natural map

$$
\mathscr{C}_{\eta}^{\mathrm{an}} \rightarrow X^{\mathrm{an}} \rightarrow \bar{\Delta}
$$

we obtain a map $\left[f^{\text {trop }}:[\rightarrow \bar{\Delta}]\right.$. By $[28$, Theorem 3.3.2], this map is seen to be a tropical stable map from a smooth tropical curve.

\subsection{Tropicalization via the Artin fan}

Let $X$ continue to denote a projective toric variety. The Artin fan of $X$ is defined to be the stack quotient $\mathscr{A}_{X}=[X / T]$. Work of Ulirsch $[37,38]$ asserts that, at the level of underlying 
topological spaces, the generic fiber of the map $\left[X \rightarrow \mathscr{A}_{X}\right]$ is canonically identified with the extended tropicalization map

$$
X^{\mathrm{an}} \rightarrow \bar{\Delta},
$$

constructed in $[26,33]$. This is made precise by the following theorem.

Given a scheme or stack $Y$ defined over a valuation ring $R$, we will use $Y_{\circ}^{\text {an }}$ to denote Raynaud's generic fiber functor applied to the formal completion of $Y$ along the maximal ideal of $R$. See $[37,38,40]$ for background on generic fibers of algebraic stacks. Note that if $Y$ is proper, the generic fiber coincides with the Berkovich analytification and will drop the $\circ$ in the subscript.

Theorem 2.12 ([38, Theorem 1.1]) There is a canonical identification of extended cone complexes given by $\mu_{\Delta}:\left|\mathscr{A}(\Delta)_{0}^{\mathrm{an}}\right| \rightarrow N(\Delta)$, making the diagram

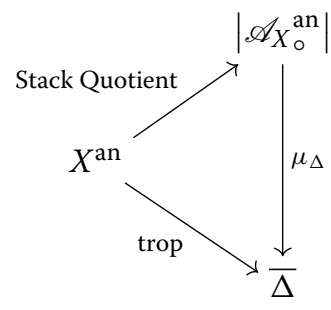

commute.

We obtain the following as an immediate corollary.

Corollary 2.13 Let $f: \mathscr{C} \rightarrow X$ be a logarithmic stable map with smooth generic fiber defined over $\operatorname{Spec}(R)$, where $R$ is a valuation ring containing $\mathbb{C}$. Let $\square$ be the skeleton of $\mathscr{C}$. Then, the tropicalization off coincides with the composite map

$$
\sqsubset \rightarrow \mathscr{C}_{\eta}^{\mathrm{an}} \rightarrow X^{\mathrm{an}} \rightarrow\left|\mathscr{A}_{X_{\circ}^{\mathrm{an}}}^{\mathrm{an}}\right|
$$

\subsection{Maps to $\mathscr{A} x$}

The purpose of this subsection is to establish a "global" version of Corollary 2.13 above. In [5], Abramovich and Wise introduce a stack of prestable logarithmic morphisms to the Artin fan $\mathscr{A}_{X}$ itself. Fixing the discrete data as before, we will use the following result of theirs.

Theorem 2.14 The stack $\mathscr{L}_{\Gamma}^{\text {pre }}\left(\mathscr{A}_{X}\right)$ is a logarithmically smooth algebraic stack, locally of finite type, and dimension $3 g-3+n$.

The reader will note that the logarithmic smoothness of this stack is in sharp contrast to the geometry of $\mathscr{L}_{\Gamma}(X)$, which satisfies Murphy's law ${ }^{2}$ in the sense of Vakil [39]. Indeed, this remarkable smoothness property was applied in [29] to show that every tropical stable map arose as the tropicalization of a family of stable maps to the Artin fan.

In [33], Thuillier defines an analytic generic fiber functor $(\cdot)^{\beth}$ from the category of schemes, locally of finite type, over trivially valued fields to analytic spaces over trivially valued fields. It should be regarded as the trivially valued version of Raynaud's generic

${ }^{2}$ Taking $X=\mathbb{P}^{n}$ with its toric boundary and $\Gamma$ to have transverse contact orders, Murphy's law for $\mathscr{L}_{\Gamma}(X)$ follows immediately from [39, Theorem M1a]. 
fiber in the nontrivially valued case. Given a scheme $X$, points of $X^{\beth}$ are, by definition, equivalence classes of maps

$$
\operatorname{Spec}(R) \rightarrow X,
$$

where $R$ is the valuation ring of a valued field extension of the trivially valued base field $k$. While the usual Berkovich analytification $X^{\text {an }}$ reflects the properness and separatedness of $X$, the analytic generic fiber is always compact and Hausdorff. This is an immediate consequence of the valuative criteria for properness and separatedness. In [37, Section 5.2], this association $X \mapsto X \beth$ is extended to algebraic stacks that are locally of finite type. We will apply this analytification functor to the stack $\mathscr{L}_{\Gamma}^{\text {pre }}(X)$.

Any point of $\mathscr{L}_{\Gamma}^{\text {pre }}(X)^{\beth}$ gives rise to a family of logarithmic prestable maps

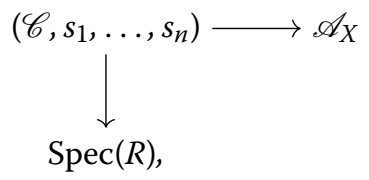

over a valuation ring extending $\mathbb{C}$. Following the same procedure as in the previous section, passing to skeletons yields a map

$$
\text { trop : } \mathscr{L}_{\Gamma}^{\mathrm{pre}}\left(\mathscr{A}_{X}\right)^{\beth} \rightarrow T_{\Gamma}^{\mathrm{pre}}(\Delta) \text {. }
$$

On the other hand, the stack $\mathscr{L}_{\Gamma}^{\text {pre }}(X)$ is a toroidal (i.e., logarithmically smooth) stack in the lisse-étale topology. There exists a continuous deformation retraction of the analytic space onto an extended cone complex:

$$
\boldsymbol{p}_{\mathscr{L}}: \mathscr{L}_{\Gamma}^{\mathrm{pre}}\left(\mathscr{A}_{X}\right)^{\beth} \rightarrow \bar{\Sigma}\left(\mathscr{L}_{\Gamma}^{\mathrm{pre}}\left(\mathscr{A}_{X}\right)\right)
$$

Note that this deformation retraction has been constructed in various closely related settings in the literature, see $[1,33]$. We briefly describe the necessary changes in the setting of Artin stacks. Let $\mathcal{X}$ be a toroidal Artin stack. Let $U \rightarrow \mathcal{X}$ be a smooth chart by a toroidal scheme without self-intersection. Consider the self-product

$$
R=U \times \mathcal{X} U \rightrightarrows U,
$$

and note that, since $\mathcal{X}$ is toroidal, the arrows above are smooth morphisms. We wish to use the toric charts of $U$ to obtain charts on $R$. This is done as follows. Given a smooth morphism $R \rightarrow U$, after shrinking, the étale local structure theorem for smooth morphisms [32, Tag 039P] yields commutative diagram

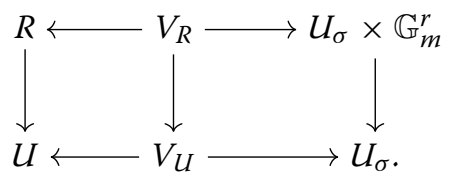

In the diagram above, $V_{R}$ and $V_{U}$ are opens, and the rightward horizontal arrows are étale. Thus, we obtain toroidal charts for $R$. The crucial point is that the torus factors in the charts for $R$ do not change the skeleton, i.e., the skeletons of $U_{\sigma}^{\beth}$ and $\left(U_{\sigma} \times \mathbb{G}_{m}^{r}\right) \beth$ are canonically identified. The extended skeleton $\bar{\Sigma}(\mathcal{X})$ of $\mathcal{X}^{\beth}$ can now be constructed via a colimit of the skeleton for the diagram $(R \rightrightarrows U)^{\beth}$. This is carried out using only cosmetic modifications to the arguments already present in the literature, see $[1,33,35,37]$. The remaining details are left to an interested reader.

The main result of this section is the relationship between the generalized extended cone complexes $T_{\Gamma}^{\text {pre }}(\Delta)$ and $\bar{\Sigma}\left(\mathscr{L}_{\Gamma}^{\text {pre }}\left(\mathscr{A}_{X}\right)\right)$. 
Theorem 2.15 There is a commutative diagram of continuous morphisms

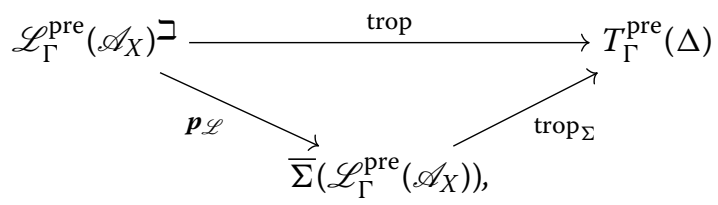

where

$$
\operatorname{trop}_{\Sigma}: \bar{\Sigma}\left(\mathscr{L}_{\Gamma}^{\mathrm{pre}}(X)\right) \rightarrow T_{\Gamma}^{\mathrm{pre}}(X)
$$

is a finite morphism of generalized extended cone complexes and becomes an isomorphism upon restriction to any cell of the source.

Proof Consider a point $p \in \mathscr{L}_{\Gamma}\left(\mathscr{A}_{X}\right)$ corresponding to a minimal logarithmic map [C $\rightarrow$ $\mathscr{A}_{X}$ ]. Suppose $p$ is contained in $W$, the locally closed stratum of the logarithmically smooth stack $\mathscr{L}_{\Gamma}\left(\mathscr{A}_{X}\right)$ parameterizing maps of combinatorial type $\Theta$. As above, there is a smooth open neighborhood $U \rightarrow \mathscr{L}_{\Gamma}\left(\mathscr{A}_{X}\right)$ containing $p$ together with an étale map

$$
U \rightarrow U_{\sigma}
$$

where $U_{\sigma}=\operatorname{Spec}\left(\mathbb{C}\left[S_{\sigma}\right]\right)$ is an affine toric variety. It follows from logarithmic smoothness of the moduli stack and the definition of the minimal log structure in [17, Section 1.4] that the monoid $S_{\sigma}$ defining $U_{\sigma}$ coincides with stalk of the minimal characteristic of $\left[C \rightarrow \mathscr{A}_{X}\right]$. Moreover, by Theorem 2.11 there is a natural identification of the cones $\sigma_{\Theta}$ and $\sigma$. Thus, the skeleton of $U^{\beth}$ is naturally identified with the extended cone $\bar{\sigma}$. The local coordinate description of the retraction map [35, Section 6] shows that the retraction

$$
U^{\beth} \rightarrow \bar{\sigma}_{\Theta}
$$

coincides with the pointwise tropicalization map construction in Sect. 2.4. Given a point of $U^{\beth}$, we obtain a family of logarithmic maps over a valuation $\operatorname{ring} \operatorname{Spec}(R)$. We may write $U^{\beth}$ as a disjoint union of sets, indexed by the locally closed $W$ stratum in $U$ to which such a family specializes. Let $\bar{\sigma}_{\Theta}^{\circ}$ be the closure of the relative interior of $\sigma_{\Theta}$ in its compactification. The skeleton $\bar{\Sigma}\left(\mathscr{L}\left(\mathscr{A}_{X}\right)\right)$ decomposes as

$$
\bar{\Sigma}\left(\mathscr{L}_{\Gamma}^{\text {pre }}\left(\mathscr{A}_{X}\right)\right)=\bigsqcup_{W} \bar{\sigma}_{\Theta_{W}}^{\circ} / \operatorname{Aut}\left(\Theta_{W}\right),
$$

where $\Theta_{W}$ is the combinatorial type associated with the generic point of the stratum $W$. Similarly, the moduli space of prestable tropical maps decomposes as

$$
T_{\Gamma}^{\text {pre }}(\Delta)=\bigsqcup_{\Theta} \bar{\sigma}_{\Theta}^{\circ} / \operatorname{Aut}(\Theta) .
$$

The skeleton $\bar{\Sigma}\left(\mathscr{L}\left(\mathscr{A}_{X}\right)\right)$ includes naturally into $\mathscr{L}_{\Gamma}^{\text {pre }}\left(\mathscr{A}_{X}\right)^{\beth}$, so by composing with the pointwise tropicalization map trop, we obtain

$$
\operatorname{trop}_{\Sigma}: \bar{\Sigma}\left(\mathscr{L}_{\Gamma}^{\mathrm{pre}}\left(\mathscr{A}_{X}\right)\right) \rightarrow T_{\Gamma}^{\mathrm{pre}}(\Delta) .
$$

By the above discussion, $\operatorname{trop}_{\Sigma}$ is an isomorphism upon restriction to any fixed cell of $\left.\bar{\Sigma}\left(\mathscr{L}_{\Gamma}\left(\mathscr{A}_{X}\right)\right)\right)$. It remains to analyze the strata of these two extended cone complexes. For this, we recall that given any logarithmic stable map $\left[f: C \rightarrow \mathscr{A}_{X}\right]$, there is a map in the category of fine but not necessarily saturated logarithmic stacks $f^{\text {us }}: C \rightarrow \mathscr{A}_{X}$ by 
the construction in $\left[28\right.$, Section 3.6]. In particular, fix an the underlying map $\left[\underline{C} \rightarrow \mathscr{A}_{X}\right]$. Given two logarithmic enhancements $f_{1}: C \rightarrow \mathscr{A}_{X}$ and $f_{2}: C \rightarrow \mathscr{A}_{X}$, the maps $f_{1}^{\text {us }}$ and $f_{2}^{\text {us }}$ coincide up to saturation. In local charts, saturation is just the normalization of a local (nonnormal) toric model. Since the normalization is finite, the saturation is finite. It follows that for each combinatorial type $\Theta$, there are finitely many strata $W$ having type $\Theta$. If $\sigma_{\Theta}$ is a cone of $T_{\Gamma}^{\text {pre }}(\Delta)$, the set of cones in $\operatorname{trop}_{\Sigma}^{-1}\left(\sigma_{\Theta}\right)$ is identified with the set of minimal logarithmic enhancements of the same underlying map, namely the map parameterized by the generic point of the stratum of type $\Theta$. In particular, this preimage is a finite union of cones, each mapping isomorphically onto $\sigma_{\Theta}$. The result follows.

We derive the first part of Theorem D as a corollary.

Corollary 2.16 There is a continuous tropicalization map

$$
\text { trop }: \mathscr{L}_{\Gamma}^{\circ}(X)^{\text {an }} \rightarrow T_{\Gamma}(\Delta),
$$

compatible with evaluation maps and forgetful maps to the moduli space of curves.

Proof Consider the sequence of maps

$$
\text { trop : } \mathscr{L}_{\Gamma}^{\circ}(X)^{\text {an }} \rightarrow \mathscr{L}_{\Gamma}(X)^{\beth} \rightarrow \mathscr{L}_{\Gamma}^{\text {pre }}\left(\mathscr{A}_{X}\right)^{\beth} \rightarrow T_{\Gamma}^{\text {pre }}(\Delta) .
$$

Since $\mathscr{L}_{\Gamma}(X)$ is proper, its formal fiber coincides with its analytification, and first map is simply the analytification of the inclusion of an open subscheme. The second arrow is constructed by applying the functor $(\cdot)^{\beth}$ for stacks to the natural map from $\mathscr{L}_{\Gamma}(X)$ to $\mathscr{L}_{\Gamma}\left(\mathscr{A}_{X}\right)$, see [37, Section 5]. The final map is the pointwise tropicalization constructed in the preceding theorem. The composition is clearly continuous, since each arrow is continuous. Forgetful morphisms to $\overline{\mathcal{M}}_{g, n}$ and evaluation maps to $X$ are all logarithmic, so functoriality follows from Theorem 2.15 above and general results on functoriality for tropicalization [35, Theorem 1.1].

As a consequence of the theorem, we rephrase the tropical lifting question as follows.

$$
\begin{gathered}
\text { When does a tropical stable map }[[\rightarrow \Delta] \text { lie in the image of the continuous map trop } \\
\text { above? }
\end{gathered}
$$

The next section takes advantage of the continuity of this map to establish the main applications.

\section{Proofs of lifting theorems}

\subsection{Polyhedrality}

Let $p=[C \rightarrow X]$ be a minimal logarithmic stable map and consider the associated map $\left[C \rightarrow \mathscr{A}_{X}\right]$. Let $U$ be a toric neighborhood of $\left[C \rightarrow \mathscr{A}_{X}\right]$ in $\mathscr{L}_{\Gamma}^{\text {pre }}\left(\mathscr{A}_{X}\right)$. Let $Z$ be the local model in the smooth topology of the moduli space $\mathscr{L}_{\Gamma}(X)$ near $p$. After possibly shrinking $Z$, the points of the compact analytic space $Z^{\beth}$ correspond to families over valuation rings of logarithmic stable maps whose special fiber, after composition with $X \rightarrow \mathscr{A}_{X}$, lies in $U$.

Let $Q$ be the stalk of the minimal characteristic monoid at $p$. As discussed in the previous section, the toric neighborhood $U$ above admits an étale map

$$
U \rightarrow \operatorname{Spec}(\mathbb{C} \llbracket Q \rrbracket) \times \mathbb{G}_{m}^{r} .
$$


The skeleton of $U^{\beth}$ is naturally identified with $\operatorname{Hom}\left(Q, \mathbb{R}_{\geq 0}\right)$. By the results of the previous section, the locus of realizable tropical curves having the combinatorial type dual to $Q$ is the image of $Z^{\beth}$ under the natural morphism

$$
Z^{\beth} \rightarrow U^{\beth} \rightarrow \operatorname{Hom}\left(Q, \mathbb{R}_{\geq 0}\right) .
$$

The latter map is the retraction map of $U^{\beth}$ onto its skeleton. Thus, on the relative interior of each cone, the image of $Z^{\beth}$ identified with the tropicalization of a subvariety of a toric variety. The fundamental theorem of tropical geometry implies that the image of $Z^{\beth}$ in the cone above is polyhedral [36, Theorem 1.1], see also [8,21,27].

Since the realizable locus is polyhedral on the relative interior of each cone, it remains to show that the image of $\mathscr{L}_{\Gamma}(X)^{\beth}$ in $T_{\Gamma}(\Delta)$ under tropicalization is topologically closed. The analytification of the moduli space of all minimal logarithmic stable maps $\mathscr{L}_{\Gamma}(X)^{\text {an }}$ is compact, since $\mathscr{L}_{\Gamma}(X)$ is proper. By continuity of the map trop constructed in the previous section, its image in $T_{\Gamma}^{\text {pre }}(\Delta)$ is compact. Since the tropical moduli space is Hausdorff, this image is also closed. A tropical curve is realizable if it is the tropicalization of a family of logarithmic maps over a valuation ring whose generic fiber carries the trivial logarithmic structure. In other words, to complete the proof we must identify the image of the locus of maps carrying trivial logarithmic structure, i.e., $\mathscr{L}_{\Gamma}^{\circ}(X)^{\text {an }}$, inside $T_{\Gamma}^{\text {pre }}(\Delta)$. Let $T_{\Gamma}^{\text {pre,o }}(\Delta)$ denote the complement of the extended faces of the generalized extended cone complex $T_{\Gamma}^{\text {pre }}(\Delta)$. We claim an equality of sets

$$
\operatorname{trop}\left(\mathscr{L}_{\Gamma}^{\circ}(X)^{\mathrm{an}}\right)=\operatorname{trop}\left(\mathscr{L}_{\Gamma}(X)^{\mathrm{an}}\right) \cap T_{\Gamma}^{\mathrm{pre}, \mathrm{o}}(\Delta)
$$

To see this, choose any family of stable maps $[\mathscr{C} \rightarrow X]$ over a valuation ring $\operatorname{Spec}(R)$ that tropicalizes to a point of $T_{\Gamma}^{\mathrm{pre}, \mathrm{o}}(\Delta)$. By definition of the latter, this means that $\mathscr{C}$ is a family of nodal curves with a skeleton such that all edge lengths are finite and thus, the generic fiber $\mathscr{C}_{\eta}$ is smooth. It follows that logarithmic structure on the base of the generic fiber of the family $\left[\mathscr{C}_{\eta} \rightarrow X\right]$ is trivial, and we obtain the set theoretic equality above. The realizable locus is thus closed in $T_{\Gamma}^{\mathrm{pre}, \mathrm{o}}(\Delta)$, which concludes the proof of Theorem $\mathrm{D}$.

Remark 3.1 The essential content of the above theorem is that the realizability conditions are given by the bend loci of the equations that describe the moduli space of maps locally. By Vakil's Murphy's law, one should expect these equations to become arbitrarily complicated, and thus one should also expect arbitrarily high complexity on the piecewise-linear side.

\subsection{Realizability of limits: Theorem A}

Let $\left[\left[_{t} \rightarrow \Delta\right]\right.$ for $t \in[0,1)$ be a continuously varying family of tropical stable maps. We have an induced moduli map

$$
[0,1) \rightarrow T_{\Gamma}(\Delta)
$$

whose image lies in the locus of realizable tropical curves. Since the realizable locus is a closed set, it follows that the limiting map $\left[\complement_{1} \rightarrow \Delta\right]$ also lies in this set, and the result follows immediately.

\subsection{Well-spacedness for good reduction}

We first recall the definition of Speyer's well-spacedness condition. Note that any genus 1 abstract tropical curve $[$ has a unique cycle, possibly a single vertex of genus 1 or a 
self-loop at a vertex. A genus 1 tropical stable map $[[\rightarrow \Delta]$ is said to be superabundant if the image of the unique cycle $L$ in $|\Delta|$ is contained in a proper affine subspace.

Definition 3.2 Let $[f:[\rightarrow \Delta]$ be a superabundant genus 1 tropical stable map. Let $H$ be a hyperplane containing the loop $L$ and consider the subgraph $\sqsubset_{H}$, the connected component of $f^{-1}(H \cap \sqsubset)$ containing $L$. Denote the 1 -valent vertices of $\complement_{H}$ by $v_{1}, \ldots, v_{k}$ and by $\ell_{i}$ the distance from $v_{i}$ to $L$. The map $f$ is well-spaced with respect to $H$ if the minimum of the multiset of distances $\left\{\ell_{1}, \ldots, \ell_{k}\right\}$ occurs at least twice.

The map $f$ is said to be well-spaced if it is well-spaced with respect to every hyperplane containing $L$.

\subsection{Proof of Theorem B}

Let $[f:[\rightarrow \Delta]$ be a tropical stable map of genus 1 such that there is a unique point $p \in \llbracket$ satisfying $g(p)=1$. In other words, the underlying graph of $\sqsubset$ is a tree. Let $\hat{\complement}_{t} \rightarrow \Delta$ be the tropical stable map obtained by replacing the genus function with one such that $g(p)=0$, and attaching a self-loop at the vertex $p$, where $t \in \mathbb{R}_{+}$is the length of this self-loop. By hypothesis, the star of $p$ in the modified map $\left[\complement_{t} \rightarrow \Delta\right]$ is realizable. By applying Speyer's genus 1 realizability theorem [31, Theorem 3.4], we see that for each value of $t>0, \hat{\complement}_{t} \rightarrow \Delta$ is realizable. Letting $t \rightarrow 0$ we obtain a continuous family of realizable tropical curves whose limit is $f$. Since the limiting map must be realizable by Theorem A, the result follows.

Remark 3.3 David Speyer has communicated to us a different approach to the proof of the above result using techniques akin to those in [31]. We record the argument here, in case it may be helpful to the reader. Let $[[\rightarrow \Delta]$ be a tropical stable map and $v \in[$ the genus 1 vertex, and assume $\operatorname{Star}(v) \rightarrow \Delta$ is realizable. Let $a_{1}, a_{2}, \ldots, a_{m}$ be the directions of the edges pointing outward from $v$ and write

$$
a_{i}=\left(a_{i 1}, a_{i 2}, \ldots, a_{i n}\right) \in \mathbb{Z}^{n} .
$$

Let $K$ be a nonarchimedean field such that the vertices of $\sqsubset$ map to points of $\Delta$ that are rational over the value group. Let $R$ be the valuation ring and $k$ the residue field. Since the link is realizable, we may build an elliptic curve $E$ over $k$ with points $z_{1}, z_{2}, \ldots z_{m} \in E(k)$ such that

$$
\sum a_{i} z_{i}=0 \in E^{n}
$$

Choose a lifting of $E$ to an elliptic curve $\mathscr{E}$ over $\operatorname{Spec}(R)$ with reduction $E$ over $k$. By the arguments of $\left[31\right.$, Section 7,8], to lift the map $[\square \rightarrow \Delta]$ we must lift the points $\left\{z_{i}\right\}$ to the generic fiber of $\mathscr{E}$ with prescribed relations, preserving $\sum a_{i} z_{i}=0$. This can be done by mimicking the calculations in Speyer's original argument, where the lifts of the points $z_{i}$ lie in the fibers of the reduction map $\mathscr{E}(R) \rightarrow E(k)$. By [30, Theorem 6.4], since we work in residue characteristic 0 , the fibers of this map are isomorphic to the additive group $R^{\times}$. The calculations are now identical to those in [31, Lemma 8.3] and the result follows.

Remark 3.4 Let $\square \rightarrow \mathbb{R}^{n}$ be a genus 1 tropical stable map as in the statement of Theorem $B$, and let $p$ be the genus 1 vertex. We have chosen to formulate the result with the hypothesis that the star of $p$ in the modified curve $\hat{\leftarrow} \rightarrow \mathbb{R}^{n}$ is realizable rather than placing a hypothesis on $\square \rightarrow \mathbb{R}^{n}$ itself. This choice has been made because the local realizability of 


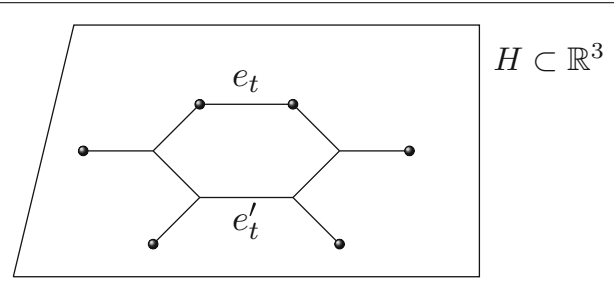

Fig. 1 A connected component of the intersection of an embedded tropical curve in $\mathbb{R}^{3}$ with a plane $H$. The black vertices indicate the points at which the cycle component leaves $H$. The edges labeled $e_{t}$ and $e_{t}^{\prime}$ have length equal to $(1-t)$

$\hat{\complement} \rightarrow \mathbb{R}^{n}$ is often easier to check, since one has an explicit coordinate on the nodal $\mathbb{P}^{1}$ in the special fiber corresponding to $p$, c.f. [15, Proposition 2.8]. One could instead impose that the star of $p$ in $\sqsubset \rightarrow \mathbb{R}^{n}$ is realizable, and the result continues to hold, as seen in the remark above.

\subsection{Proof of Theorem C}

For the reader's benefit, a "proof-by-picture" is given in Fig. 1. Let $[[\rightarrow \Delta]$ be a genus 1 tropical curve whose cycle is contained in a hyperplane $H$. Assume that this map is well-spaced. Furthermore, assume that the minimum of the distances in Definition 3.2 is 0 and occurs exactly twice. It follows that there exist two vertices $v_{1}$ and $v_{2}$, belonging to the cycle $L$, where $\left[\right.$ leaves $H$. Choose a path in $L$ between $v_{1}$ and $v_{2}$ and any family of tropical curves $\left[\left[_{t} \rightarrow \Delta\right]\right.$ for $t \in[0,1)$ such that the distance between $v_{1}$ and $v_{2}$ is $(1-t)$. Observe that for each value of $t<1$, the tropical map $\left[\left[_{t} \rightarrow \Delta\right]\right.$ is well-spaced and thus realizable by [31, Theorem 3.4]. However, in the limiting map, $\sqsubset_{1} \rightarrow \Delta$, there is a unique point on $L$ at which the cycle $L$ leaves the hyperplane $H$. It follows that the limiting map cannot be well-spaced. However, the map is realizable by Theorem A.

\section{Acknowledgements}

During the preparation of this paper, I have benefited greatly from conversations with friends and colleagues including Dori Bejleri, Renzo Cavalieri, Dave Jensen, Sam Payne, Martin Ulirsch, and Ravi Vakil. I would like to thank David Speyer, in particular, for sharing his insights on realizability in the good reduction case, see Remark 3.3. I would also like to thank Dan Abramovich for pointing out a gap in an earlier version of this paper. During important phases of the project I was a student at Brown University and Yale University, and it is a pleasure to acknowledge these institutions here. The final version of this document was greatly improved as a result of the comments of two anonymous referees. Final revisions were completed, while the author was a member at the Institute for Advanced Study in Spring 2017. This research was partially supported by NSF Grant CAREER DMS-1149054 (PI: Sam Payne).

Received: 23 September 2016 Accepted: 27 February 2017

Published online: 01 June 2017

\section{References}

1. Abramovich, D., Caporaso, L., Payne, S.: The tropicalization of the moduli space of curves. Ann. Sci. Éc. Norm. Supér. 48, 765-809 (2015)

2. Abramovich, D., Chen, Q.: Stable logarithmic maps to Deligne-Faltings pairs II. Asian J. Math. 18, 465-488 (2014)

3. Abramovich, D., Chen, Q., Gillam, D., Huang, Y., Olsson, M., Satriano, M., Sun, S.: Logarithmic geometry and moduli. In: Farkas, G., Morrison, I. (eds.) Handbook of moduli: Volume I. Advanced Lectures in Mathematics (ALM), vol. 24. International Press, Somerville, MA. ISBN 978-1-57146-257-2/pbk (2013)

4. Abramovich, D., Chen, Q., Marcus, S., Ulirsch, M., Wise, J.: Skeletons and fans of logarithmic structures. In: Nonarchimedean and Tropical Geometry. Based on Two Simons Symposia, Island of St. John, March 31-April 6, 2013 and Puerto Rico, February 1-7, 2015, pp. 287-336. Springer, Cham (2016)

5. Abramovich, D., Wise, J.: Invariance in logarithmic Gromov-Witten theory. arXiv:1306.1222 (2013)

6. Baker, M., Payne, S., Rabinoff, J.: On the structure of non-Archimedean analytic curves. In: Tropical and Nonarchimedean geometry, vol. 605 of Contemp. Math., pp. 93-121. Amer. Math. Soc., Providence, RI (2013)

7. Baker, M., Payne, S., Rabinoff, J.: Nonarchimedean geometry, tropicalization, and metrics on curves. Algebr. Geom. 3 63-105 (2016) 
8. Bieri, R., Groves, J .R.: The geometry of the set of characters iduced by valuations. J. Reine Angew. Math. (Crelle's Journal) 347, 168-195 (1984)

9. Cavalieri, R., Hampe, S., Markwig, H., Ranganathan, D.: Moduli spaces of rational weighted stable curves and tropical geometry. In: Forum of Mathematics, Sigma, vol. 4 (2016)

10. Cavalieri, R., Markwig, H., Ranganathan, D.: Tropicalizing the space of admissible covers. Math. Ann. 364, 1275-1313 (2016)

11. Cavalieri, R., Markwig, H., Ranganathan, D.: Tropical compactification and the Gromov-Witten theory of $\mathbb{P}^{1}$. Sel. Math. arXiv:1410.2837 (to appear)

12. Chan, M.: Topology of the tropical moduli spaces $\bar{M}_{2, n}$. arXiv:1507.03878 (2015)

13. Chan, M., Galatius, S., Payne, S.: The tropicalization of the moduli space of curves II: topology and applications. arXiv:1604.03176 (2016)

14. Chen, Q.: Stable logarithmic maps to Deligne-Faltings pairs I. Ann. Math. 180, 341-392 (2014)

15. Cheung, M.-W., Fantini, L., Park, J., Ulirsch, M.: Faithful realizability of tropical curves. Int. Math. Res. Not., rnv269 (2015)

16. Fulton, W., Sturmfels, B.: Intersection theory on toric varieties. Topology 36, 335-353 (1997)

17. Gross, M., Siebert, B.: Logarithmic Gromov-Witten invariants. J. Am. Math. Soc. 26, 451-510 (2013)

18. Jensen, D., Ranganathan, D.: Brill-Noether theory for curves of a fixed gonality. arXiv:1701.06579 (2017)

19. Katz, E.: Lifting tropical curves in space and linear systems on graphs. Adv. Math. 230, 853-875 (2012)

20. Len, Y., Ranganathan, D.: Enumerative geometry of elliptic curves on toric surfaces. arXiv:1510.08556 (2015)

21. Maclagan, D., Sturmfels, B.: Introduction to Tropical Geometry. Volume 161 of Graduate Studies in Mathematics. American Mathematical Society, Providence, RI (2015)

22. Mikhalkin, G.: Enumerative tropical geometry in $\mathbb{R}^{2}$. J. Am. Math. Soc 18, 313-377 (2005)

23. Nishinou, T.: Correspondence theorems for tropical curves. arXiv:0912.5090 (2009)

24. Nishinou, T.: Describing tropical curves via algebraic geometry. arXiv:1503.06435 (2015)

25. Nishinou, T., Siebert, B.: Toric degenerations of toric varieties and tropical curves. Duke Math. J. 135, 1-51 (2006)

26. Payne, S.: Analytification is the limit of all tropicalizations. Math. Res. Lett. 16, 543-556 (2009)

27. Popescu-Pampu, P., Stepanov, D.: Local tropicalization. In: Brugallé, E., Cueto, M.A., Dickenstein, A., Feichtner, E-M., Itenberg, I. (eds.) Algebraic and combinatorial aspects of tropical geometry. Proceedings based on the CIEM workshop on tropical geometry, International Centre for Mathematical Meetings (CIEM), Castro Urdiales, Spain, December 12-16, 2011. Contemporary Mathematics, vol. 589. American Mathematical Society (AMS), RI. ISBN 978-0-8218-91469/pbk (2013)

28. Ranganathan, D.: Skeletons of stable maps I: Rational curves in toric varieties. J. Lond. Math. Soc. arXiv:1506.03754 (2015) (to appear)

29. Ranganathan, D.: Superabundant curves and the Artin fan. Int. Math. Res. Not. (2016)

30. Silverman, J.: The Arithmetic of Elliptic Curves, Applications of Mathematics. Springer, Berlin (1986)

31. Speyer, D.E.: Parameterizing tropical curves. I: curves of genus zero and one. Algebra Number Theory 8, 963-998 (2014)

32. The Stacks Project Authors: Stacks project. http://stacks.math.columbia.edu (2017)

33. Thuillier, A.: Géométrie toroïdale et géométrie analytique non archimédienne. Application au type d'homotopie de certains schémas formels. Manuscr. Math. 123, 381-451 (2007)

34. Tyomkin, l.: Tropical geometry and correspondence theorems via toric stacks. Math. Ann. 353, 945-995 (2012)

35. Ulirsch, M.: Functorial tropicalization of logarithmic schemes: the case of constant coefficients. arXiv:1310.6269 (2013)

36. Ulirsch, M.: Tropical compactification in log-regular varieties. Math. Z. 280, 195-210 (2015)

37. Ulirsch, M.: Non-archimedean geometry of Artin fans. arXiv:1603.07589 (2016)

38. Ulirsch, M.: Tropicalization is a non-archimedean analytic stack quotient. Math. Res. Lett. arXiv:1410.2216 (to appear)

39. Vakil, R.: Murphy's law in algebraic geometry: badly-behaved deformation spaces. Invent. Math. 164, 569-590 (2006)

40. Yu, T.Y.: Tropicalization of the moduli space of stable maps. Math. Z. 281, 1035-1059 (2015)

41. Yu, T.Y.: Gromov compactness in non-archimedean analytic geometry, J. Reine Angew. Math. (Crelle's Journal). arXiv:1401.6452 (to appear)

\section{Submit your manuscript to a SpringerOpen ${ }^{\circ}$ journal and benefit from:}

- Convenient online submission

- Rigorous peer review

- Immediate publication on acceptance

- Open access: articles freely available online

- High visibility within the field

- Retaining the copyright to your article

Submit your next manuscript at $\mathbf{s p r i n g e r o p e n . c o m ~}$ 